\title{
Recursos de calidad en internet para calcular el índice $h$
}

\author{
Sandra MARTORELL \\ Universidad Politécnica de Valencia \\ sanferm2@epsg.upv.es
}

\begin{abstract}
Resumen:
Este artículo habla del indicador bibliométrico propuesto por Hirsch conocido como índice $h$, el cual permite medir el éxito de un investigador a lo largo de su carrera a partir de dos parámetros, la relación entre la producción científica de un autor y las citas que dicha producción recibe por parte de otros autores.

En primer lugar se hará una presentación del medidor para posteriormente hablar de aquellos recursos en internet que nos dan unas garantías de calidad para poder calcularlo de forma fiable y rigurosa, siendo éstos los que ofrecen las bases de datos Web of Knowledge y Scopus.
\end{abstract}

Palabras clave: índice $h$; bibliometría; Web of Knowledge; Scopus; Google Scholar.

\section{Quality Internet resources to calculate the H-index}

\begin{abstract}
:
This article is about the H-index, bibliometric indicator suggested by Hirsch that measures the success of a researcher along his/her professional career from two parameters: the link connecting the scientific production of a particular author and the quotes about this production by other authors. First of all, we will introduce this indicator followed by the Internet resources to guarantee its quality in order to calculate the H-index in a reliable and rigorous way, as for Web of Knowledge and Scopus databases.
\end{abstract}

Key Words: H-index; bibliometrics; Web of Knowledge; Scopus; Google Scholar.

\section{Referencia normalizada:}

Martorell, S. (2014): Recursos de calidad en internet para calcular el índice h. Historia y Comunicación Social. Vol. 19. Núm. Especial Marzo. Págs. 615-627.

Sumario: 1. Introducción. 1.2. Estado de la cuestión. 2. Metodología. 3. WOK y Scopus, dos bases de datos para calcular el índice $h$. 3.1. Índice $h$ en WOK. 3.2. Índice $h$ en Scopus. 4. Conclusiones. 5. Agradecimientos. 6. Referencias bibliográficas. 


\section{Introducción}

Las cifras legitiman. Lo vemos en las ciencias experimentales, pero también en las ciencias sociales, las humanidades, las artes y en la vida en general. Necesitamos datos numéricos que avalen las decisiones de los gobiernos, el grado de una discapacidad, el coeficiente intelectual o la magnitud de una catástrofe. Porque lejos de guiarnos por otros parámetros seguimos confiando (erróneamente o no) en que las cifras confieren un valor objetivo a aquello sobre lo que se aplican. Así pues creemos que éstas son capaces de medir incluso el éxito y la calidad profesional de académicos y científicos, no en vano se han creado medidores para tal efecto, como es el caso del índice $h$, un indicador bibliométrico que, aunando dos variables (el número de publicaciones y el número de citas recibidas), nos ofrece una cifra que revela el éxito y prestigio de un autor entre sus colegas, lo cual se convierte en un aval indirecto de la calidad de sus trabajos.

El uso de este indicador es cada vez más habitual, y son numerosos los programas y recursos en internet para calcularlo. Ahora bien, no todos son fiables ni nos dan los mismos resultados, con el añadido de que calculándolo en algunos de ellos la cifra de $h$ se puede manipular a conveniencia. Ésta es la razón de ser de esta propuesta que más allá de hacer una revisión teórica del índice $h$, plantea los problemas de determinados recursos de cálculo como Google Scholar Citations (en español, Citas de Google Académico) y propone los que, atendiendo a cuestiones de calidad y rigor serían los más adecuados, siendo éstos los de Web of Knowledge (Thomson Reuters) y Scopus (Elsevier). Con éstos dos últimos se realizará el cálculo con el fin de ver cómo funcionan y la diferencia de resultados que se obtienen con cada uno ellos, lo cual nos dará una confirmación del valor relativo del indicador y nos advertirá de la necesidad de seguir trabajando para conseguir una formulación más precisa y fidedigna.

\subsection{Estado de la cuestión}

El índice $h$ fue propuesto en 2005 por Jorge E. Hirsch, y se caracteriza por aunar en una única magnitud el número de publicaciones de un autor y la cantidad de citas que se han hecho a tales publicaciones, midiendo así la visibilidad, la calidad y la cantidad de la producción científica de éste. En relación a la calidad cabe aclarar que es algo indirecto; se entiende que si un autor es citado repetidamente por otros autores entendidos en la materia es porque consideran que tiene valor aquello que plantea, por tanto son ellos mismos los que, mediante la citación están legitimando su trabajo considerándolo de referencia.

Según Hirsch (2005: 16.569), "un autor tendrá un índice $h$ si el $h$ de sus $N p$ trabajos tienen al menos $h$ citas cada uno y los otros $N p$ - $h$ trabajos no tienen más de $h$ citas cada uno", o dicho en palabras de Rousseau (en Salgado y Páez, 2007:180) "el Índice de Hirsch es $h$ si $h$ es el rango más alto (número natural más alto) tal que las primeras $h$ publicaciones reciben cada una al menos $h$ citas" (2006:23). 
Para obtener el índice $h$ podemos crear dos columnas. En la primera ordenamos de menor a mayor los diferentes trabajos de un autor $(1,2,3, \ldots)$, y en la segunda las citas recibidas de mayor a menor. Habrá un punto en que ambas columnas coincidan en la misma cifra, ésa será $h$.

Viene a ser lo mismo que Hirsch plantea en el gráfico 1. En él vemos dos ejes, el horizontal representa la cantidad de artículos y el vertical el número de citas por artículo. El punto donde la línea discontinua cruza la de 45 grados es lo que determina $h$.

\section{Gráfico 1}

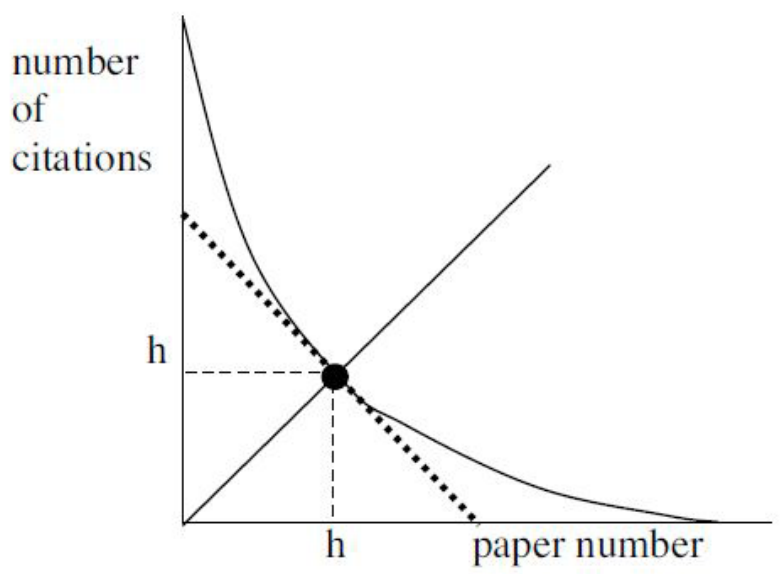

Como vemos es un índice sencillo de calcular que según el Grupo Scimago (2006:304) "tiende a valorar un esfuerzo científico prolongado a lo largo de toda la vida académica”.

Ahora bien, el índice $h$ también tiene inconvenientes. Se puede convertir en un problema a la hora de usarlo como criterio de selección entre investigadores de campos diferentes, ya que es, como definieron Bornmann y Marx (2011:77), campodependiente.

Esto significa que cada campo tiene sus propias características en lo que a productividad y citación se refiere. Por ejemplo hay disciplinas cuyos grupos de investigadores son más grandes porque hay mayor tendencia a trabajar en equipo como ocurre más comúnmente en las ciencias experimentales y su producción de artículos es mayor que en materias de carácter más teórico como las pertenecientes a las humanidades en las que se da en mayor medida el trabajo individual. Esto pone de manifiesto la poca imparcialidad que supondría si investigadores de humanidades y ciencias experimentales tuvieran de competir entre ellos considerándose únicamente como criterio de decisión el índice $h$. 
Lo mismo ocurre cuando comparamos a un autor de una disciplina más dada a la publicación continua y rutinaria de artículos con otro de una disciplina que tiende más a la publicación de libros (como ocurre de forma más habitual dentro de las humanidades), algo que se lleva a cabo de forma más espaciada en el tiempo. Ante estas situaciones advertimos una clara desventaja de los investigadores pertenecientes a campos de índole más teórica, lo que pone en evidencia el sesgo que se daría y por consiguiente el defecto en la objetividad de medidor. Es por ello por lo que no deberían compararse investigadores de campos diferentes ya que no estarían en igualdad de condiciones.

A parte de ésta hay otras limitaciones del índice $h$ que no pasan por alto en los estudios de autores como Costas y Bordons (2007ab), Bornmann y Marx (2011) u otros como Dorta-González y Dorta-González (2010) o Silva Ayçaguer (2012) tal y como pasamos a ver a continuación:

- Perjudica a los investigadores con una trayectoria más corta y por tanto con menos publicaciones y la correspondiente citación a éstas, dado que esto aumenta con el tiempo, algo que también afirmaban Kelly y Jennions (2006:168). Esto afecta de forma considerable a los colectivos más jóvenes que aun no han dispuesto del tiempo necesario para forjar una carrera sólida y dilatada que es de lo que en parte depende el índice $h$. Algo que ya fue advertido por el propio Hirsch, que propuso como solución dividir $h$ entre los años de carrera del investigador ( $\mathrm{h} / \mathrm{n}$; es lo que se conoce como cociente $m$ ), aunque también existe la posibilidad de hacer el cálculo únicamente para un periodo de tiempo concreto.

- Ni sirve únicamente tener muchos artículos publicados si no han sido citados, ni sirve tener pocos artículos pero con muchas citas, sino que siempre se ha de dar una relación compensada de ambos parámetros.

- Es muy complicado medir el índice $h$ con precisión, teniendo en cuenta que la automatización del proceso de cálculo puede tener errores debido a lo difícil que resulta compilar información para llevarlo a cabo. Uno de los más comunes es la incapacidad de las herramientas de cálculo a la hora de discernir entre autores llamados del mismo modo, o cuando se trata de reconocer a los que siendo los mismos firman o aparecen citados de dos formas diferentes. Por otro lado, si la medición del índice $h$ se hace de forma manual, podemos llegar a conseguir un resultado más preciso pero entonces se pierde el valor de la de la agilidad en el cálculo, especialmente cuando estamos hablando de autores con una vasta trayectoria o con muchos artículos o citas que referencian a los mismos.

- Las autocitas pueden manipular los resultados. En este sentido cabe realizar algunos matices. De entrada la autocita como tal no supone un aspecto negativo, ya que un investigador puede haber publicado sobre un tema anteriormente y estar justificada, la cual, dentro de este contexto es perfectamente 
legítima. El conflicto viene cuando hay una tendencia habitual e injustificada a la autocita alentada por el interés de contribuir a aumentar el valor de $h$.

- Otra forma de manipulación se consigue mediante la creación de documentos falsos que son considerados como válidos por algunas herramientas de cálculo del índice $h$, tal y como puede ser Google Scolar Citations, asunto que trataremos más adelante.

Como vemos es un medidor bastante controvertido, aunque no por ello poco considerado. De hecho son muchos los que los se han aventurado a hacer propuestas de mejora o alternativas pero ninguno ha conseguido tener la fuerza y la aceptación suficiente para desbancar o al menos complementar asiduamente al índice $h$.

\section{Metodología}

El sistema metodológico que hemos propuesto parte de planteamientos teóricos así como de análisis prácticos para llevar a cabo las correspondientes conclusiones. Para esto último, el primer paso a realizar ha consistido en realizar una selección de recursos en línea para calcular el índice $h$, de entre los cuales podemos discernir entre dos tipos fundamentalmente. Por un lado aquéllos que son inherentes a una base de datos, es decir, que son ofrecidos por éstas. Por otro, aquéllos que son complementos o programas independientes pero que para el cálculo toman la información de determinadas bases de datos siendo la más común para ello Google Scholar (conocido en español como Google Académico). Entre los recursos de este tipo tenemos complementos como el que creó Giovambattista Ianni para el navegador Firefox, el Scholar H-index Calculator, el Scholarometer o el popular programa Publish or Perish (publica o muere), también de acceso abierto desarrollado por Anne-Wil Harzing, la cual lanzó su primera versión en 2006 (éste usa tanto la información de Google Scholar, como desde su versión 4.1, la de Microsoft Academic Search). No obstante para el presente trabajo nos hemos centrado en los que ofrecen las principales bases de datos de contenidos académicos y científicos por varios motivos, entre ellos porque al ser bases de datos de peso suelen contar con herramientas que se encuentran en constante evolución y mejora, sin correr tanto el riesgo de perecer tal y como ha ocurrido con recursos como scHcolar index, creado por Nicolas Roussel, y que actualmente se encuentra en desuso por problemas de acceso a la información de Google Scholar. Además se trata de recursos avalados por la utilización de los usuarios.

En este sentido hemos hecho una selección de las tres bases de datos más populares para este cometido, siendo éstas Web of Knowledge, en adelante WOK (Thomson Reuters), Sciverse / Scopus (Elsevier) y Google Scholar (Google Inc.), de entre las cuales finalmente hemos descartado Google Scholar, y con ella el recurso de cálculo que ofrece, Google Scholar Citations. Éste, lanzado el 20 de julio de 2011, permite a los investigadores darse de alta e ir configurando su perfil. La herramienta propone un listado de documentos encontrados que el autor decide si agregar o descartar (en 
caso de que no le correspondan), así como añadir otros manualmente que se le hayan pasado por alto al buscador.

De Google Scholar la creadora de Publish or Perish defendía que abría las puertas a la "democratización" del análisis de citas (Harzing y van der Wal, 2008:72). Como ella otros hablaron de su utilidad y valor, algo que se da esencialmente por los motivos siguientes:

- Está al servicio de la comunidad de internautas

- Es de carácter gratuito.

- Contempla otros idiomas "en pie de igualdad al inglés", algo que constituye "un rasgo encomiable en la medida que permite escapar del sesgo que supone la discriminativa hegemonía idiomática del inglés" (Silva Ayçaguer, 2013).

- Tiene en consideración un abanico más amplio de recursos como puedan ser conferencias, informes técnicos, eprints (Whitton, 2013:1), u otros como tesis doctorales.

No obstante, también cuenta con inconvenientes:

- Presenta algunos problemas para refinar la búsqueda. Ya no hablamos solamente cuando se trata de autores con los mismos apellidos y las mismas iniciales, sino en ocasiones, solamente con los mismos apellidos.

- Muchas veces no es capaz de diferenciar los documentos meramente académicos de los que no lo son.

- Gran presencia de trabajos duplicados en los resultados, debido generalmente a que hay diferentes versiones de un mismo artículo que Google Scholar no ha sido capaz de reagrupar, por lo que aparecen como trabajos diferentes; por ejemplo, un trabajo en su versión preprint publicada en la web del autor y su versión final publicada en una revista científica (Torres-Salinas, Ruiz-Pérez y Delgado-López-Cózar, 2009:508).

Ahora bien, de todos los inconvenientes los que más nos preocupan son los relativos a la calidad de los contenidos que es el motor que ha impulsado el presente artículo. Entre ellos destacamos el más relevante, que consideramos que avala nuestra decisión de excluir los recursos ofrecidos por Google Scholar a la hora de calcular el índice $h$.

Desde el punto de vista de la calidad, es importante que haya un control de lo que se indexa. Para ello hay métodos diversos, entre los que se podría encontrar el de revisión por pares (conocido en inglés como peer review), que si bien también cuenta con algunos inconvenientes, es uno de los sistemas de validación más usados por la comunidad científica y académica.

En este sentido las bases de datos como WOK o Scopus sí llevan un control riguroso, algo que acredita su nobleza, a diferencia de Google Scholar que mezcla los contenidos de calidad que han sido validados por algún método de revisión como el 
peer review con otros que no han pasado por ningún filtro de calidad, llevando a cabo una inclusión de citas indiscriminada.

Es este sentido es especialmente escandaloso el caso de los documentos falsos que Google Scholar considera como legítimos, algo que deja al descubierto la vulnerabilidad de la base de datos para ser manipulada. Estos documentos falsos pueden ser creados mediante programas como pueda ser SCIgen, que Google Scholar no es capaz de distinguir de los artículos legítimos y los incluye en igualdad de condiciones, algo que afecta al rigor de la base de datos a la hora de calcular el índice $h$. Un ejemplo de ello es el conocido caso de Ike Antkare, un científico falso creado por Cyril Labbé en 2010, que gracias a este programa y a la facilidad para ser incluido en el cálculo de Google Scholar, consiguió convertirse en un periodo récord de tiempo en uno de los científicos más citados de la historia.

Casos de esta índole son lo suficientemente contundentes para descartar a Google Scholar y su Citas de Google Scholar, en la búsqueda de unos resultados de calidad.

Así pues nos hemos decantado por los recursos de cálculo que ofrecen únicamente WOK y Scopus, y hemos procedido a hacer una comparativa a partir del cálculo del índice $h$. Cabe decir que ambas son de pago, lo cual si a priori podría considerarse una desventaja, para el tema que nos ocupa no tiene mayor relevancia ya que lo que prima como apuntábamos es la calidad, y en ese aspecto son dos bases de datos de acreditada solvencia y prestigio a la hora de valorar cuantitativamente a los investigadores.

\section{WOK y scopus, dos bases de datos para calcular el índice H}

Una vez hecha la selección de recursos para la obtención de forma automática de $h$ vamos a ver cómo se lleva a cabo el proceso de cálculo en cada una de las bases de datos. Cabe tener en cuenta que no son recursos idénticos sino que cada base de datos tiene su propia indexación de contenidos, lo cual nos permitirá ver las diferencias en el resultado final que pueden surgir entre ambas.

Para llevar a cabo la prueba hemos tomado de muestra al teórico en estudios filmicos David Bordwell, catedrático de la Universidad de Wisconsin y una referencia internacional en su área de estudio.

\section{1. Índice $H$ en $W O K$}

Tras acceder a la página de WOK, y desde el buscador de todas las bases de datos (All Databases), introducimos el primer apellido del autor y la inicial de su nombre.

Una vez le damos a buscar tenemos una siguiente pantalla donde acotaremos más la búsqueda. Entonces le indicaremos que se encuentra dentro de artes y humanidades y que su área de investigación se incluiría dentro de cine, radio y televisión. 
En lo que refiere al tipo de documentos centraremos la búsqueda en "article" y "review", aunque cabe advertir de que esto en este caso no afectará a la cifra final del índice $h$.

Pulsamos en el texto "Create Citation Report" (gráfico 2), ubicado en la parte superior derecha.

Gráfico 2

Sort by: Publication Date - newest ti $\overrightarrow{ }$

IIII Create Citation Report

Y automáticamente nos ofrece los resultados tal y como vemos en el gráfico 3.

Gráfico 3

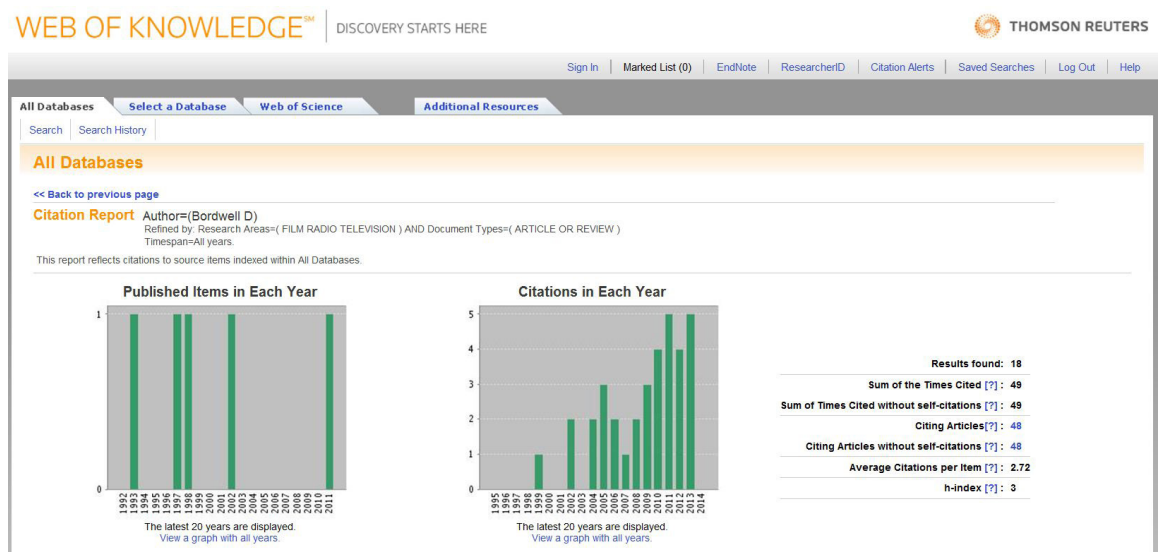

Para $W O K$ el índice $h$ de David Bordwell es 3, dato que se complementa como se puede ver con otras informaciones tales como las suma de las veces citado, la misma suma excluyendo las autocitas o la media de citas por ítem, entre otros, así como con la puesta a disposición del usuario de dos gráficos: uno correspondiente a los ítems publicados por año y otro a las citas recibidas también por años.

Además como apunta el gráfico 4 podemos obtener información de cada publicación de forma independiente sólo con desplazarnos en la web hacia abajo. Esto nos permite ver de cada ítem las citas por año así como el total. Y siguiendo con la calidad, si prestamos atención a la publicación a la cual pertenece cada artículo comprobaremos que se trata de las más importantes en la materia, algo que pone de 
manifiesto la consideración que $W O K$ tiene a la hora de hacer la selección de datos a diferencia de la descartada Google Scholar.

\section{Gráfico 4}

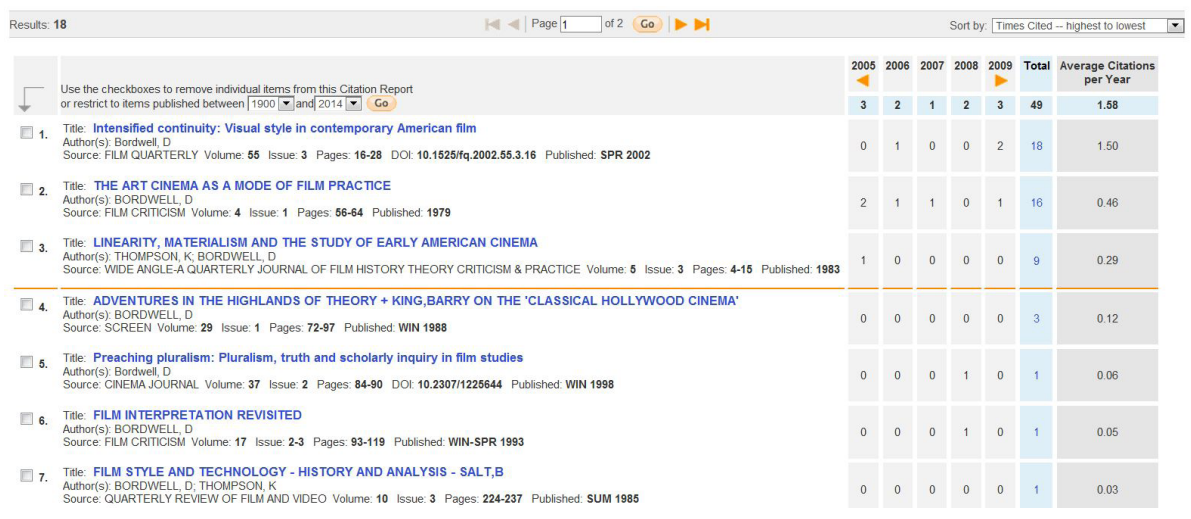

\section{2. Índice $H$ en Scopus}

Procedemos ahora a llevar a cabo la obtención de $h$ esta vez desde Scopus; de igual modo que hemos hecho en $W O K$ accedemos a la base de datos, y nos situamos en la pestaña de búsqueda de autores (Author search), donde ingresaremos en un campo el apellido y en otro la inicial del nombre. Además acotaremos el área desde ahí mismo a las humanidades y las ciencias sociales descartando las otras tres opciones que vienen marcadas por defecto.

En este caso Scopus ha encontrado en base a los datos introducidos a dos autores cuyo nombre y apellido coincide (ver gráfico 5). Ante esto tendremos que seleccionar el correcto, algo que es especialmente útil para los casos en que dos autores se llaman igual pero son distintos. En nuestro caso, tenemos que comprobarlo, para lo cual usamos la pestaña "show last title" para ver si el título de la publicación que nos ofrece corresponde al teórico que buscamos. 


\section{Gráfico 5}

\begin{tabular}{|c|c|c|}
\hline \multirow[t]{2}{*}{$\begin{array}{l}\square \text { All } \\
\square \text { Page }\end{array}$} & \multicolumn{2}{|c|}{$\begin{array}{l}\text { With selected: } \\
\text { 自 Show documents I } \mid W \text { View citation overview I Request to merge authors }\end{array}$} \\
\hline & ors & Documents \\
\hline \multicolumn{2}{|c|}{$\frac{1}{1}$ Bordwell, David } & $\begin{array}{l}\text { 目 } 12 \\
\text { 드 Show Last Title }\end{array}$ \\
\hline \multicolumn{2}{|c|}{ Bordwell, David } & $\begin{array}{l}\text { 目 } 3 \\
\text { ㄷ Hide Last Title } \\
\text { Most recent document title: } \\
\text { Space and narrative in the films of ozu1 }\end{array}$ \\
\hline $\begin{array}{l}\nabla \text { All } \\
\nabla \text { Page }\end{array}$ & \multicolumn{2}{|c|}{ 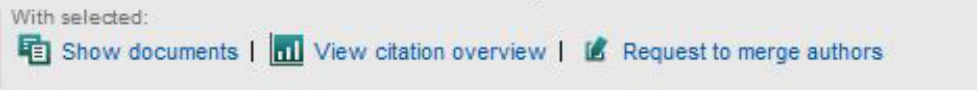 } \\
\hline
\end{tabular}

Display $20 \quad \nabla$ results per page

En relación al autor del que teníamos dudas (el segundo) hemos visto el título del último documento y comprobado que sí pertenece a quien buscamos, con lo que en este caso, ambos autores referirían a la misma persona, por lo que marcamos sendas casillas y seleccionamos "View citation overview". Vemos el resultado en el gráfico 6:

\section{Gráfico 6}

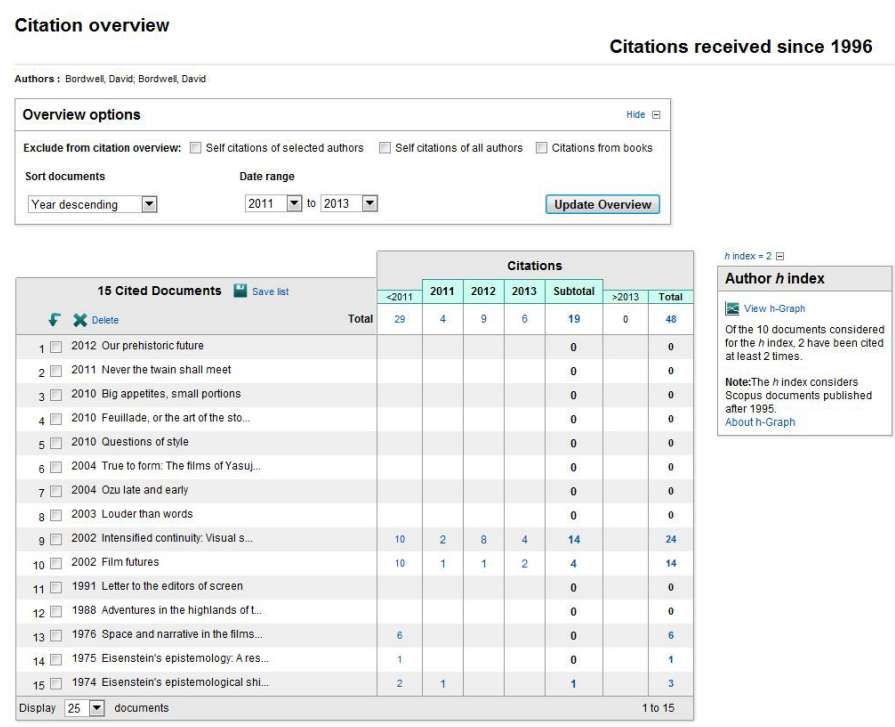


También vemos que nos da información por títulos y por años, aunque en general nos da menos información adicional que $W O K$. No obstante, lo que más llama la atención es que la cifra correspondiente a $h$ diverge respecto a la obtenida en la otra base de datos, siendo en vez del 3 anterior, un 2, algo que pone de manifiesto las diferencias entre ambas.

Esto se debe a que los datos entre los que ahondar en una y en otra son diferentes, tanto por la cantidad como por el tipo. Ya hemos hablado de que en Scopus hay más publicaciones de carácter internacional, mientras que en $W O K$ hay cierto sesgo hacia las publicaciones en inglés, entre otros asuntos. Por lo demás no están tan lejos la una de la otra en cuanto a la calidad de los contenidos que indexan o el tiempo que se tarda en calcular el valor de $h$, limitándose esto último a escasos minutos.

\section{Conclusiones}

En la actualidad todo se mide, lo cual explica el crecimiento de la bibliometría. Lo apuntaba Egghe (2007), y se ha puesto de manifiesto en la cantidad de medidores bibliométricos que han aparecido en los últimos tiempos para evaluar numéricamente la actividad y la calidad profesional de los científicos en base a una serie de parámetros.

En este sentido y atendiendo a las palabras de Dorta-González y Dorta-González (2010) "existe consenso en admitir que los artículos son el resultado más directo de la investigación, especialmente cuando se consideran aquellas revistas en las que existe un sistema de selección que garantiza la calidad y originalidad de los trabajos". Y dentro de los artículos se presta especial atención a la citación, no en vano ésta, para bien o para mal, repercute a la hora de tomar decisiones en asuntos de asignación de subvenciones o contratación de personal entre otros (Bakkalbasi et al., 2006).

Así pues tanto los artículos como las citas han servido para evaluar a los investigadores. Hoy estos dos parámetros se contemplan en un único indicador popularizado en los últimos tiempos, el conocido como índice $h$.

A pesar de las variantes y complementos que se han propuesto para corregir y subsanar las no pocas limitaciones que presenta, el índice $h$ sigue manteniendo cierta hegemonía dada su sencillez, motivo por el cual hemos centrado nuestro trabajo en él y en los recursos que ofrece internet para poder calcularlo de forma automática en pocos minutos.

De todas las opciones de la red nos hemos quedado con la alternativa que nos proponen WOK y Scopus. Esto se debe a que estamos hablando de la calidad en Internet y éstas dos bases de datos son las que, en la carrera por indexar las publicaciones más rigurosas y más cualificadas, han sabido obtener mayor prestigio internacional. 
No obstante, al obtener el valor de $h$ en ambas nos encontramos con que esta cifra puede variar de una a la otra, con lo que comprobamos que no ofrecen resultados exactos sino más bien orientativos. Lo mismo que ocurre con el índice $h$; su popularidad ha hecho que se tome como una fórmula infalible cuando en realidad no tendría que servir de única referencia, sino que sería preferible combinar diferentes indicadores, ya que la investigación en sí es un proceso multidimensional que difícilmente se puede abordar desde una única dimensión, tal y como desde hace tiempo vienen advirtiendo autores como Bornmann y Marx (2011:78), Costas y Bordons (2007b:428) o Martin (1996:345) entre otros.

Todo esto nos hace cuestionarnos si realmente la calidad puede medirse cuantitativamente. Las teorías que barajamos sobre ello son escépticas pero lo cierto es que independientemente de sesgos personales la tendencia se dirige a hacer que esto sea posible tal y como hemos podido aventurar en el presente artículo que, ante tal coyuntura, ofrece los recursos necesarios para saciar la información a través de una cifra controvertida pero enormemente aclamada.

\section{Agradecimientos}

La investigación para la realización de este artículo fue llevada a cabo con el apoyo del proyecto de investigación "Estudio y análisis para el desarrollo de una red de conocimiento sobre estudios fílmicos a través de plataformas web 2.0", financiado por el Plan Nacional de I+D+i del Ministerio de Economía y Competitividad español (código HAR2010-18648).

\section{Bibliografía}

BAKKALBASI, N.; BAUER, K.; GLOVER, J.; WANG, L. (2006). "Three options for citation tracking: Google Scholar, Scopus and Web of Science". En: Biomedical Digital Libraries. [12-06-2013] http://www.bio-diglib.com/content/pdf/17425581-3-7.pdf

BORNMANN, L.; MARX, W. (2011). "The $h$ index as a research performance indicator". En: European Science Editing, 37(3), 77-80.

COSTAS, R.; BORDONS, M. (2007a). "The h-index: advantages, limitations and its relation with other bibliometric indicators at the micro level". En: Journal of Informetrics 1, 193-203.

COSTAS, R.; BORDONS, M. (2007b). "Una visión crítica del índice h: algunas consideraciones derivadas de su aplicación práctica". En: El profesional de la información, 16(5), 427-432. 
DORTA-GONZÁLEZ, P; DORTA-GONZÁLEZ, M. I. (2010). «Indicador bibliométrico basado en el índice h». En: Revista Española de Documentación Científica, $33,225-245$.

EGGHE, L (2007). "From $\mathrm{h}$ to g: the evolution of citation indices", en Research Trends. [13-05-2013]. http://www.researchtrends.com/issue1-september-2007/ from-h-to-g/.

GRUPO SCIMAGO (2006). "El índice h de Hirsch: aportaciones a un debate". En: El profesional de la información, 15(4), 304-306.

HARZING, A.W.: VAN DER WAL, R. (2008). "Google Scholar as a new source for citation analysis". En: Ethics in Science and Environmental Politics, 8, 61-73.

HIRSCH, J. E. (2005). "An index to quantify an individual's scientific research output". En: Proceedings of the National Academy of Sciences of the United States of America, 102, 16.569-16.572.

KELLY, C. D.; JENNIONS, M. D. (2006). "The h-index and career assessment by numbers". En: Trends in Ecology and Evolution, 21(4), 167-170.

MARTIN, B. R. (1996). "The use of multiple indicators in the assessment of basic research". En: Scientometrics, 36(3), 343-362.

ROUSSEAU, R. (2006). "New developments related to the Hirsch index". En: Science Focus, 1(4), 23-25.

SALGADO, J. F.; PÁEZ, D. (2007). «La productividad científica y el índice h de Hirsch de la psicología social española: convergencia entre indicadores de productividad y comparación con otras áreas». En: Psicothema, 19(2), 179-189.

TORRES-SALINAS, D.; RUIZ-PÉREZ, R.; DELGADO-LOPEZ-CÓZAR, E. (2009). "Google Scholar como herramienta para la evaluación científica". En: El profesional de la información, 18(5), 501-510.

SILVA AYÇAGUER, L.C. (2012). «El Îndice-h y Google académico: una simbiosis cienciométrica inclusiva». En: REVISTA CUBANA DE INFORMACIÓN EN CIENCIAS DE LA SALUD (ACIMED), 23(3).

WHITTON, M. (2013). "Finding your h-index (Hirsch index) in Google Scholar". En: University of Southampton Library Factsheet, 3, 1-2.

\section{La autora}

Sandra Martorell es doctoranda en la Universidad Politécnica de Valencia, donde se licenció en Comunicación Audiovisual tras haber realizado parte de sus estudios en la Universidad La Lumière de Lyon (Francia) y la Universidad Pompeu Fabra de Barcelona. Combina su trabajo de periodista con la investigación en materia de comunicación, especialmente en la que refiere a las redes sociales en Internet. 